\title{
Tecnura
}

\section{Caracterización fisicoquímica de cuarzo en el municipio de La Playa de Belén, departamento de Norte de Santander}

\author{
Physiochemical characterization of quartz in the municipality \\ of La Playa de Belén-North Santander department
}

\author{
Lina Marcela Hoyos Palacio*, Edwin Espinel Blanco**, Yeixon Quintero Martínez***
}

Fecha de recepción: 15 de septiembre de 2014

Fecha de aceptación: 6 de abril de 2015

Como citar: Hoyos Palacio, L. M., Espinel Blanco, E., \& Quintero Martínez, Y. (2015). Caracterización fisicoquímica de cuarzo en el municipio de La Playa de Belén, departamento de Norte de Santander. Revista Tecnura, 19(45), 41-51. doi: 10.14483/udistrital.jour.tecnura.2015.3.a03

\section{RESUMEN}

El cuarzo es un silicato, se presenta en una gran variedad de condiciones geológicas, aparece en muchas rocas ígneas y metamórficas, y es un constituyente principal de pegmatitas graníticas. Entre los minerales, el cuarzo es el más próximo a un compuesto químico puro y tiene propiedades físicas constantes. Debido a sus aplicaciones y usos, se convierte en un material que contribuye a mejorar las condiciones de vida del hombre, por tal razón se hace evidente la necesidad de conocer sus propiedades para proponer aplicaciones óptimas. Este trabajo describe las propiedades físicas y químicas del cuarzo en el municipio de La Playa de Belén, departamento de Norte de Santander, Colombia, mediante el uso de técnicas elementales. Se utilizan las técnicas de Microscopía Electrónica de Barrido (MEB) con analizador de energías dispersas (EDS), difracción de rayos $X(D R X)$, análisis termogravimétrico (TGA) y diferencial termogravimétrico (DTG). Con este análisis se obtuvieron las características composicionales, químicas, morfológicas y se evaluó el comportamiento térmico de acuerdo con la composición.

Palabras clave: caracterización, cuarzo, fisicoquímicas, materiales, propiedades.

\begin{abstract}
Quartz is a silicate, available in a variety of geological conditions, it appears in many igneous and metamorphic rocks, and it is a major constituent of granite pegmatite. Among minerals, quartz is the closest to a pure chemical compound and has constant physical properties, and due to their applications and uses it becomes a material that contributes to improving the living conditions of man. For that reason, it is necessary to know their properties to propose optimal applications. This work presents the physicochemical characterization of quartz in the municipality of La Playa de Belen (North Santander Department, Colombia) using elementary techniques through studies to establish the physical
\end{abstract}

* Ingeniera química, doctora en Ingeniería y Termodinámica. Coordinadora de la Línea de Nanotecnología y Biomateriales, Universidad Pontificia Bolivariana, Medellín, Colombia. Contacto: lina.hoyos@upb.edu.co

** Ingeniero mecánico, magíster en Ingeniería. Investigador del grupo GITYD, Universidad Francisco de Paula Santander, Seccional Ocaña, Ocaña, Colombia. Contacto: eeespinelb@ufpso.edu.co

*** Ingeniero mecánico. Investigador del grupo GITYD, Universidad Francisco de Paula Santander, Seccional Ocaña, Ocaña, Colombia. Contacto: yquinterom@ufpso.edu.co 
and chemical properties of this mineral. Techniques such as scanning electron microscopy (SEM) with disperse energy analyzer (EDS), X-ray diffraction (XRD), thermogravimetric (TGA) and differential thermogravimetric analysis (DTG) were used. The compositional, chemical and morphological characteristics are obtained, and the thermal behavior is evaluated according to the composition.

Keywords: characterization, materials, physicochemical, properties, quartz.

\section{INTRODUCCIÓN}

Los minerales son materiales que conforman las rocas de la corteza terrestre, son los que más abundan y están formados por silicatos, óxidos y otros compuestos de oxígeno, como carbonatos en cantidades mínimas (Mendoza, Baron Gonzalez, \& Parra Vargas, 2007). Las diferentes asociaciones de minerales caracterizan las rocas ígneas, sedimentarias y metamórficas, y así como las venas minerales, las pegmatitas, las rocas meteorizadas y los suelos, tienen la facultad de dar información sobre las condiciones ambientales en que se formaron, sus componentes, la determinación de propiedades químicas y físicas. El suelo está constituido en gran parte por silicatos; los ladrillos, las piedras, el cemento y el vidrio empleados en la construcción son silicatos o se derivan de estos y son los materiales cerámicos más importantes (Cornellis, Cornelius, \& Hurbult, 1996).

Existen otras razones por las cuales se deben estudiar los silicatos: el silicio es el elemento más abundante después del oxígeno, y el óxido de silicio, $\mathrm{SiO}_{2}$, es el más abundante de todos los óxidos, siendo el cuarzo la forma más conocida (Holmes, 1980). Este compuesto forma una estructura ordenada regular y una irregular que se llama vidrio de cuarzo o sílice (Alipour \& Denoyel, 2011). El cuarzo se emplea en aparatos ópticos y científicos, en chips de silicio, en la producción de vidrio (Anderson, 1998), es además la materia prima de artesanías, utensilios domésticos, producción de porcelanas, vidrios y objetos refractarios (Shackelford, 2010), tallado en lentes y prismas. A los usos ya mencionados se une su utilización como material de relleno en la preparación de resinas compuestas o composites dentales (Veranes, 2003), escoria activada alcalinamente autoclave mezclada con polvo de cuarzo (Rashad, 2012).

Los estudios sobre las propiedades y aplicaciones del cuarzo a través de técnicas elementales han permitido comprender este material (Leal, Cortona, \& Pagan, 2005), la evolución de la cementación y la historia de la formación basada de isótopos de oxígeno en los cubrimientos de cuarzo (Hyodo, Kozdon, Pollington, \& Valley, 2014), así como su aplicación en la técnica de deflexión de microbalanza de cristal de cuarzo y haz de sonda electroquímica combinado en disolventes eutécticas profundas (Hilman, Ryder, Zaleski, Ferreira , Beasley, \& Vieil, 2014), entre otras muchas aplicaciones que tienen este mineral.

La caracterización fisicoquímica del cuarzo se realiza para conocer las propiedades de mayor relevancia de este mineral. Para esto se empleó una metodología descriptiva, el diseño de un plan de muestreo y técnicas elementales para la caracterización de materiales, las cuales permitieron conocer su composición química y morfológica; además se evaluó el comportamiento térmico de acuerdo con la composición.

\section{METODOLOGÍA}

\section{Ubicación}

La zona donde se extrajeron las muestras se encuentra ubicada en el municipio de La Playa de Belén, departamento de Norte de Santander, Colombia, a una altitud de 1.754 metros sobre el nivel del mar, bajo las coordenadas N 08¹1'13,2" y W 07316’22,3”, con una temperatura promedio de $21^{\circ} \mathrm{C}$. 


\section{Selección de muestras}

La selección de las muestras en el área de estudio se realizó por medio del diseño de un muestreo estratificado, como lo muestra la Tabla 1. Este método consiste en dividir el área de estudio en estratos sin que estos solapen o interfieran entre sí (Alfaro, 2002), de esta manera se logra obtener muestras representativas que permiten conocer las características del conjunto de rocas estudiadas. Para garantizar que todas las muestras tengan la misma posibilidad de ser elegidas, se estableció dividir el área de estudio en cuatro estratos, cada uno dividido a su vez en tres secciones; por cada sección se tomaron muestras de tres kilogramos, de las cuales se seleccionaron muestras al azar para comprobar la variabilidad o uniformidad.

El tamaño de la muestra tomada de campo se redujo al tamaño de estudio del laboratorio. Se realizó de acuerdo con el procedimiento definido en la Norma Técnica Colombiana NTC 3674, la cual describe las prácticas para la reducción del tamaño de las muestras de agregados, tomadas en campo, para realizar ensayos. De acuerdo con la norma se eligió trabajar con el Método B cuarteo.
Las muestras se redujeron en cantidad y tamaño, por lo cual se utilizó una porra para impactar las rocas y fragmentarlas hasta obtener partículas del tamaño de 75 micras. Además se utilizó un mortero de porcelana para macerar el mineral y conseguir partículas homogéneas.

\section{Determinación del porcentaje de humedad}

Para determinar la humedad de las muestras de cuarzo se adaptó la norma mexicana NMX-L159-SCFI-2003, la cual especifica el procedimiento para determinar las propiedades de la barita empleada en fluidos de perforación. Se utilizó el mineral en polvo con un tamaño de grano de $75 \mu \mathrm{m}$ pasante del tamiz número 200 . Se usó una cantidad de 80 gramos de masa, medidos en una balanza digital marca Pioneer Ohaus Corp. Pinebrook, con una precisión de 0,05 gramos. Se vertió el mineral en recipientes secos de vidrio, se introdujeron en un horno digital de precisión marca Pinzuar Ltda., modelo PG 190, a una temperatura de $\pm 110{ }^{\circ} \mathrm{C}$ por un tiempo de 24 horas.

Tabla 1. Diseño del muestreo estratificado

\begin{tabular}{|c|c|c|}
\hline Estrato & Nombre de la muestra & Coordenada \\
\hline \multirow{3}{*}{$E_{1}$} & $M_{1}$ & $(0,0)$ \\
\hline & $\mathrm{M}_{2}$ & $(2.5,4)$ \\
\hline & $M_{3}$ & $(5,0)$ \\
\hline \multirow{3}{*}{$E_{2}$} & $\mathrm{M}_{4}$ & $(0,0)$ \\
\hline & $M_{5}$ & $(2.5,4)$ \\
\hline & $M_{6}$ & $(5,0)$ \\
\hline \multirow{3}{*}{$\mathrm{E}_{3}$} & $M_{7}$ & $(0,0)$ \\
\hline & $M_{8}$ & $(2.5,4)$ \\
\hline & $M_{9}$ & $(5,0)$ \\
\hline \multirow{3}{*}{$\mathrm{E}_{4}$} & $M_{10}$ & $(0,0)$ \\
\hline & $M_{11}$ & $(2.5,4)$ \\
\hline & $M_{12}$ & $(5,0)$ \\
\hline
\end{tabular}

Fuente: elaboración propia. 


\section{Determinación de la densidad}

Esta propiedad del mineral se determinó por medio de la adecuación de la norma técnica colombiana NTC 221, la cual establece el método para establecer la densidad del cemento hidráulico. El material fue secado durante 24 horas a $100{ }^{\circ} \mathrm{C}$, se pesó cada muestra de 63 gramos de tamaño de grano del pasante en tamiz número $200(75 \mu \mathrm{m})$, de acuerdo con el procedimiento descrito en la norma.

\section{Análisis termogravimétrico y diferencial termogravimétrico}

Las condiciones que se utilizaron para este caso de estudio son las siguientes: atmósfera oxidante de $\mathrm{O} 2$ (aire), la velocidad lineal de calentamiento fue de $10{ }^{\circ} \mathrm{C} / \mathrm{min}$ y el límite máximo de temperatura de $700{ }^{\circ} \mathrm{C}$.

\section{Microscopía Electrónica de Barrido (MEB), con analizador del Espectrómetro de Energías Dispersas (EDS)}

El recubrimiento de las muestras se realizó en un equipo marca Denton Vacuum, y se utilizó recubrimiento de oro. El cuarzo fue adherido al soporte con cinta de grafito. El microscopio que se usó para analizar las muestras es marca Jeol JSM-6490LV.

\section{Difracción de rayos $X$}

Para el empleo de la técnica de difracción de rayos x se utilizó un equipo marca Panalytical, cuya referencia es X'pert PRO MPD. El tipo de radiación empleada fue radiación de cobre con una longitud de onda característica lambda $(\lambda)$ de 1,5406 angstrom $(\AA)$. El barrido se realizó entre 6 y 70 grados, el paso fue de 0,013 grados y el tiempo por paso de 59 segundos. El tubo fue polarizado con una potencia de 1,8 kW (40 mAmp, 45kV).

\section{RESULTADOS}

\section{Humedad}

El método se basa en el cálculo de la humedad higroscópica, que se realiza para determinar el cambio de masa del mineral mediante un proceso de secado a una temperatura constante en un horno. Al ser calentadas las muestras se elimina el agua libre contenida en los espacios porosos dentro de las partículas constituyentes (NMX-L-159-SCFI, 2003), se compara la masa inicial de la muestra y la final luego del secado, definido en la ecuación

Tabla 2. Contenido de humedad de las muestras de cuarzo

\begin{tabular}{cccc}
\hline Número muestra & Masa inicial (gramos) & Masa final (gramos) & \% de humedad \\
\hline$M_{1}$ & 80 & 79,93 & 0,0875 \\
\hline$M_{2}$ & 80 & 79,94 & 0,0750 \\
\hline$M_{3}$ & 80 & 79,97 & 0,0375 \\
\hline$M_{4}$ & 80 & 79,95 & 0,0625 \\
\hline$M_{5}$ & 80 & 79,95 & 0,0625 \\
\hline$M_{6}$ & 80 & 79,94 & 0,0750 \\
\hline$M_{7}$ & 80 & 79,98 & 0,0250 \\
\hline$M_{8}$ & 80 & 79,98 & 0,0250 \\
\hline$M_{9}$ & 80 & 79,96 & 0,0500 \\
\hline$M_{10}$ & 80 & 79,97 & 0,0375 \\
\hline$M_{11}$ & 80 & 79,96 & 0,0500 \\
\hline$M_{12}$ & 80 & 79,98 & 0,0250 \\
\hline
\end{tabular}

Fuente: elaboración propia. 
(1). Los cálculos obtenidos con esta práctica se resumen en la tabla 2.

$$
\% \text { humedad }=\frac{m-m_{s}}{m} \times 100
$$

Donde,

$m$ : es la masa inicial de la muestra, en gramos $g$. $m_{2}$ : es la masa de la muestra seca, en gramos $g$.

La humedad determinada por la práctica es menor que $0,1 \%$, lo cual garantiza que las muestras estudiadas presentan bajo contenido de agua. Esto se debe a que los enlaces que unen el mineral son considerados de tipo iónico en el $50 \%$ y covalente en el otro $50 \%$, lo que hace del cuarzo un mineral con enlaces químicos fuertes y por esta característica no lo afecta.

\section{Densidad}

El método usado permitió determinar la densidad específica de las muestras de cuarzo. La práctica de encontrar la densidad de un mineral consiste en establecer la relación entre una cantidad determinada de masa y el volumen del líquido desplazado por dicha cantidad de masa en el frasco de
Le Chatelier y se establece en la ecuación (2). Los valores de densidad encontrados se resumen en la Tabla 3.

$\rho$ : Densidad calculada del cuarzo $\left(\mathrm{g} / \mathrm{cm}^{3}\right)$.

$$
\rho=\frac{m}{v_{d}}
$$

$m$ : es la masa de la muestra de cuarzo en gra$\operatorname{mos}(\mathrm{g})$.

$v_{d}$ : es el volumen desplazado $\left(\mathrm{cm}^{3}\right)$.

La densidad de las muestras de cuarzo encontrada fue de $2,65 \mathrm{~g} / \mathrm{cm}^{3}$ con $\pm 0,01 \mathrm{~g} / \mathrm{cm}^{3}$, que es igual al valor teórico estandarizado para el material de $2,65 \mathrm{~g} / \mathrm{cm}^{3}$, lo cual evidencia la pureza encontrada en la composición química y la calidad del mineral.

\section{Análisis termogravimétrico y diferencial termogravimétrico}

La técnica usada permite comparar los termogramas obtenidos con datos teóricos y de esta manera determinar la presencia o no de materiales diferentes al cuarzo. En la figura 1 se observan los termogramas para las muestras 1, 4, 7 y 11 .

Tabla 3. Densidad específica de las muestras de cuarzo

\begin{tabular}{cccc}
\hline Muestra & Masa (gramos) & Volumen desplazado $\left.\mathbf{( c m}^{\mathbf{3}}\right)$ & Densidad $\mathbf{~} / \mathbf{c m}^{\mathbf{3}}$ \\
\hline $\mathrm{M}_{1}$ & 63 & 23,8 & 2,65 \\
\hline $\mathrm{M}_{2}$ & 63 & 23,8 & 2,65 \\
\hline $\mathrm{M}_{3}$ & 63 & 23,9 & 2,64 \\
\hline $\mathrm{M}_{4}$ & 63 & 23,7 & 2,66 \\
\hline $\mathrm{M}_{5}$ & 63 & 23,8 & 2,65 \\
\hline$M_{6}$ & 63 & 23,8 & 2,65 \\
\hline$M_{7}$ & 63 & 23,8 & 2,65 \\
\hline$M_{8}$ & 63 & 23,9 & 2,64 \\
\hline$M_{9}$ & 63 & 23,9 & 2,64 \\
\hline$M_{10}$ & 63 & 23,9 & 2,64 \\
\hline$M_{11}$ & 63 & 23,9 & 2,64 \\
\hline$M_{12}$ & 63 & 23,9 & 2,64 \\
\hline
\end{tabular}

Fuente: elaboración propia. 

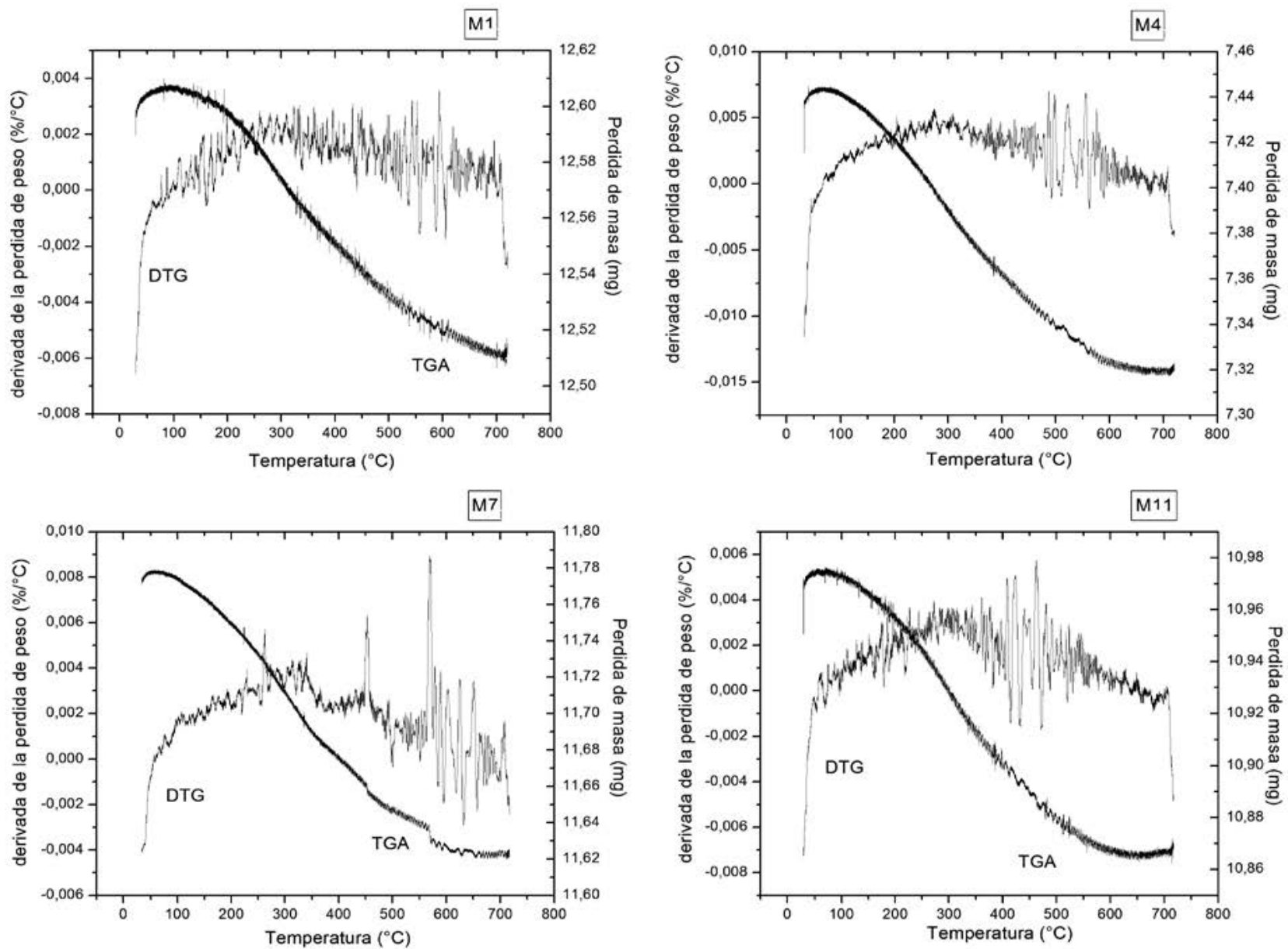

Figura 1. Termogramas de las muestras $1,4,7$ y 11 de cuarzo. En ellas se observa el comportamiento con el cambio de temperatura en el análisis termogravimétrico (TGA ) y el cambio de fase de cuarzo- $\alpha$ a cuarzo- $\beta$ alrededor de la temperatura de $573{ }^{\circ} \mathrm{C}$ en el análisis diferencial termogravimétrico (DTG).

Fuente: elaboración propia.

Los termogramas permiten observar que alrededor de los $573{ }^{\circ} \mathrm{C}$ se presenta altas variaciones en las curvas pertenecientes a la derivada de la pérdida de masa (DTG). Esto se debe al cambio de fase cristalina de cuarzo- $\alpha$ a cuarzo- $\beta$, transición de tipo desplazativo que indica que el volumen de la muestra aumenta debido al cambio en las dimensiones de la red cristalina sin que se presente ruptura de los enlaces. Todos los termogramas presentan variación en la pérdida de masa y su derivada en el rango de temperatura comprendido entre $60{ }^{\circ} \mathrm{C}$ y $260{ }^{\circ} \mathrm{C}$ debido a la deshidratación de los silicatos y a la pérdida de peso.

\section{Microscopía Electrónica de Barrido (MEB), con analizador del Espectrómetro de Energías Dispersas (EDS)}

En la figura 2 se presentan las micrografías con las cuales se evaluó las características morfológicas para cada una de las muestras observadas a 500 $X$. Las imágenes permitieron comprobar el tipo de fractura concoidal o en capas característica del cuarzo. Asimismo se observa la forma irregular de los cristales, un aspecto importante para la influencia si se utiliza como material industrial por 


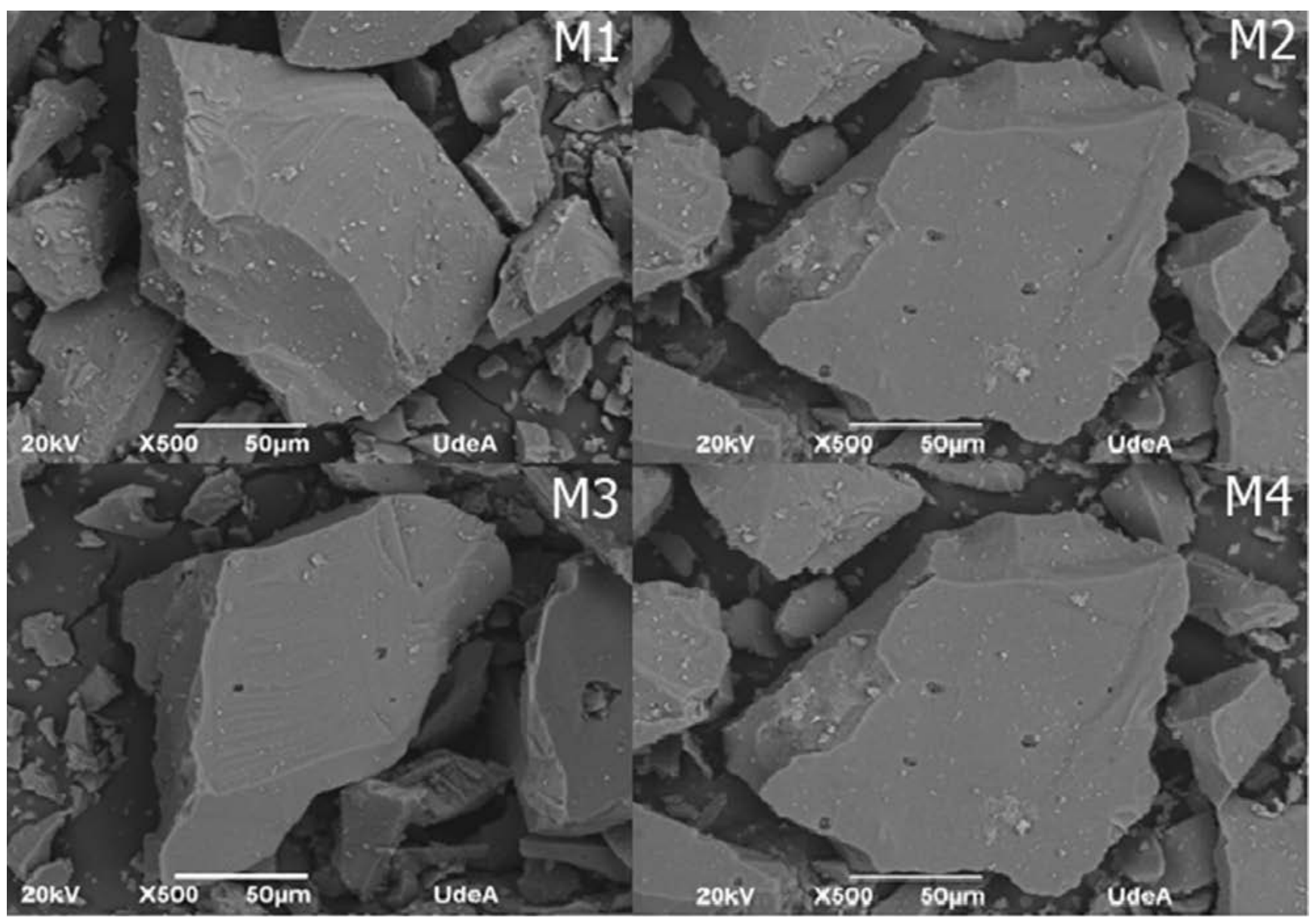

Figura 2. Micrografía a $500 \mathrm{X}$ de las muestras $1,4,6$ y 8 de las muestras de cuarzo. Se observa la forma irregular de los cristales.

Fuente: elaboración propia.

la unión mecánica que se produce si se aglomera con otros compuestos.

En las micrografías a $100 \mathrm{X}$ que se muestran en la figura 3 se observa que las partículas son irregulares en su forma y homogéneas la distribución de tamaño de grano, lo cual permite verificar y mejorar las apreciaciones de acuerdo con la superficie de los cristales y con ello la calidad del material porque se observa la superficie irregular y alisada característica del cuarzo. Asimismo, se observa que existe una gran distribución de partículas que van desde tamaños de $1 \mu \mathrm{m}$ hasta mayores de $75 \mu \mathrm{m}$.

Con la finalidad de conocer la composición química de las muestras, se analizaron con una microsonda de dispersión energética, lo cual permitió determinar la composición química de las muestras de cuarzo (tabla 4). Se encontró que todas las muestras son puras, excepto la primera, en la cual aparece una pequeña inclusión de hierro de $1,05 \%$ del peso total de la muestra debido a la contaminación durante la trituración.

La composición química del cuarzo corresponde a un mineral de alta pureza por su contenido único de $\mathrm{SiO}_{2}$, que cumple con los requerimientos de calidad industriales tales como la cristalería, componentes ópticos, electrónico y como materia prima para la obtención de sílice fundida (Leal, Cortona, \& Pagan, 2005). 


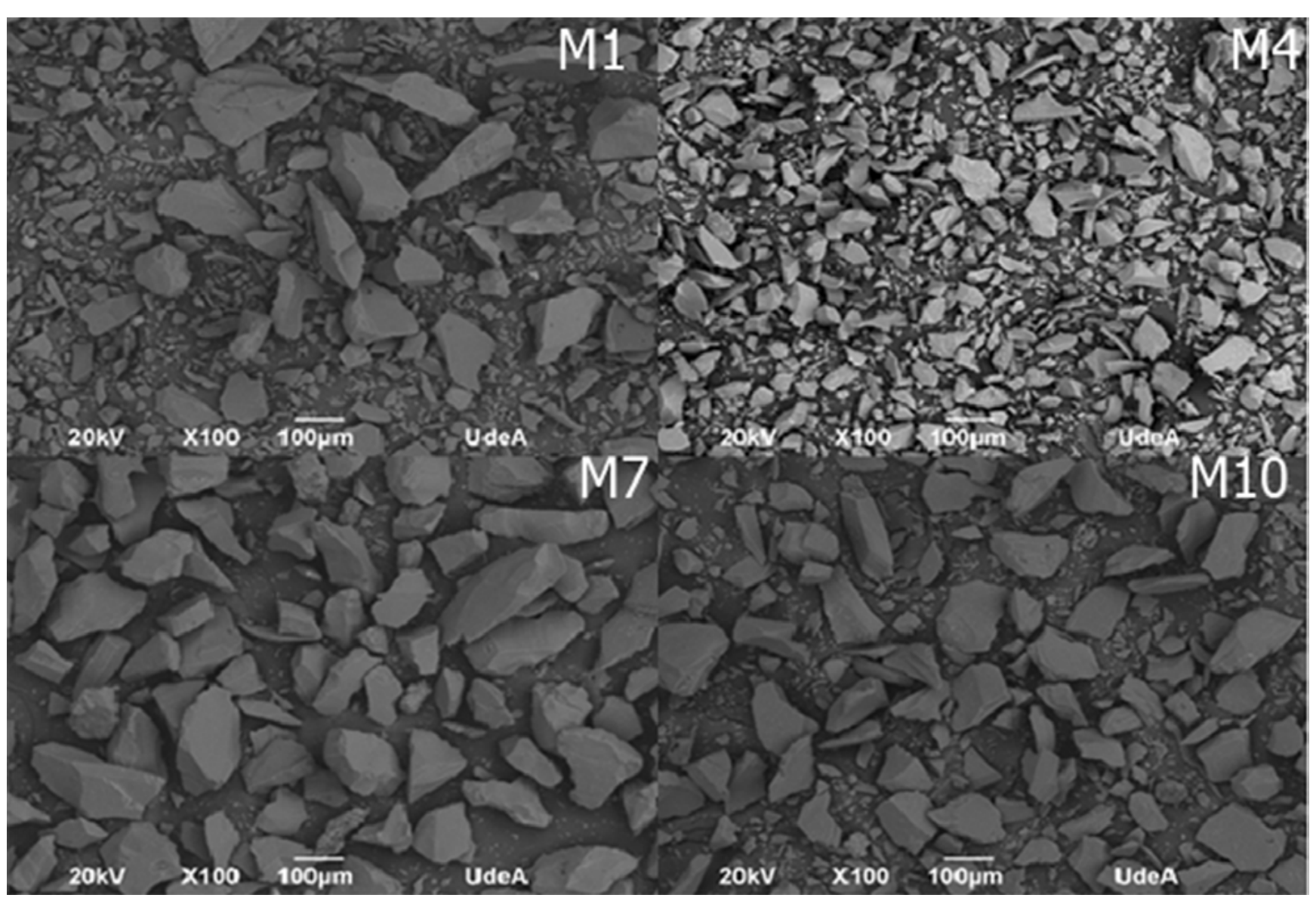

Figura 3. Micrografía a $500 \mathrm{X}$ de las muestras de cuarzo 1, 4, 7 y 10. Se observa la forma irregular de los cristales y su distribución.

Fuente: elaboración propia.

Tabla 4. Composición química del cuarzo determinado por el espectrómetro de energías dispersas (EDS)

\begin{tabular}{cccccccc}
\hline & \% EN PESO & \multicolumn{3}{c}{ \% ATÓMICO } & \% & \\
\hline MUESTRA & $\mathbf{O}$ & $\mathbf{S i}$ & $\mathbf{F e}$ & $\mathbf{O}$ & $\mathbf{S i}$ & $\mathbf{F e}$ & TOTAL \\
\hline$M_{1}$ & 52,56 & 46,39 & 1,05 & 66,29 & 33,33 & 0,38 & 100 \\
\hline$M_{2}$ & 54,56 & 45,95 & & 67,37 & 32,63 & & 100 \\
\hline$M_{3}$ & 55,99 & 44,01 & & 69,07 & 30,93 & & 100 \\
\hline$M_{4}$ & 50,87 & 49,13 & & 64,51 & 35,49 & & 100 \\
\hline$M_{5}$ & 52,07 & 47,93 & & 65,60 & 34,40 & & 100 \\
\hline$M_{6}$ & 52,66 & 47,34 & & 66,13 & 33,87 & & 100 \\
\hline$M_{7}$ & 53,88 & 46,12 & & 67,22 & 32,78 & & 100 \\
\hline$M_{8}$ & 55,82 & 44,18 & & 68,92 & 31,09 & & 100 \\
\hline$M_{9}$ & 56,93 & 43,07 & & 69,88 & 30,12 & & 100 \\
\hline$M_{10}$ & 56,94 & 43,06 & & 69,89 & 30,11 & & 100 \\
\hline$M_{11}$ & 55,19 & 44,81 & & 68,37 & 31,63 & & 100 \\
\hline$M_{12}$ & 55,19 & 44,81 & & 68,37 & 31,63 & & 100 \\
\hline
\end{tabular}

Fuente: elaboración propia. 


\section{Difracción de rayos $X$}

Las intensidades encontradas en la difracción de rayos $\mathrm{X}$ se compararon con las descritas en el Manual de Mineralogía (Dana \& Hurlbut, 1960). Se comprobó que las intensidades corresponden al mismo valor del intervalo angular $2 \theta$, como se puede observar en los difractogramas de la figura 4, en la cual se aprecia que la única fase presente es del cuarzo, y complementa los resultados obtenidos mediante la técnica del espectroscopio de energías dispersas, en el cual se determinó la alta pureza de las muestras de cuarzo.
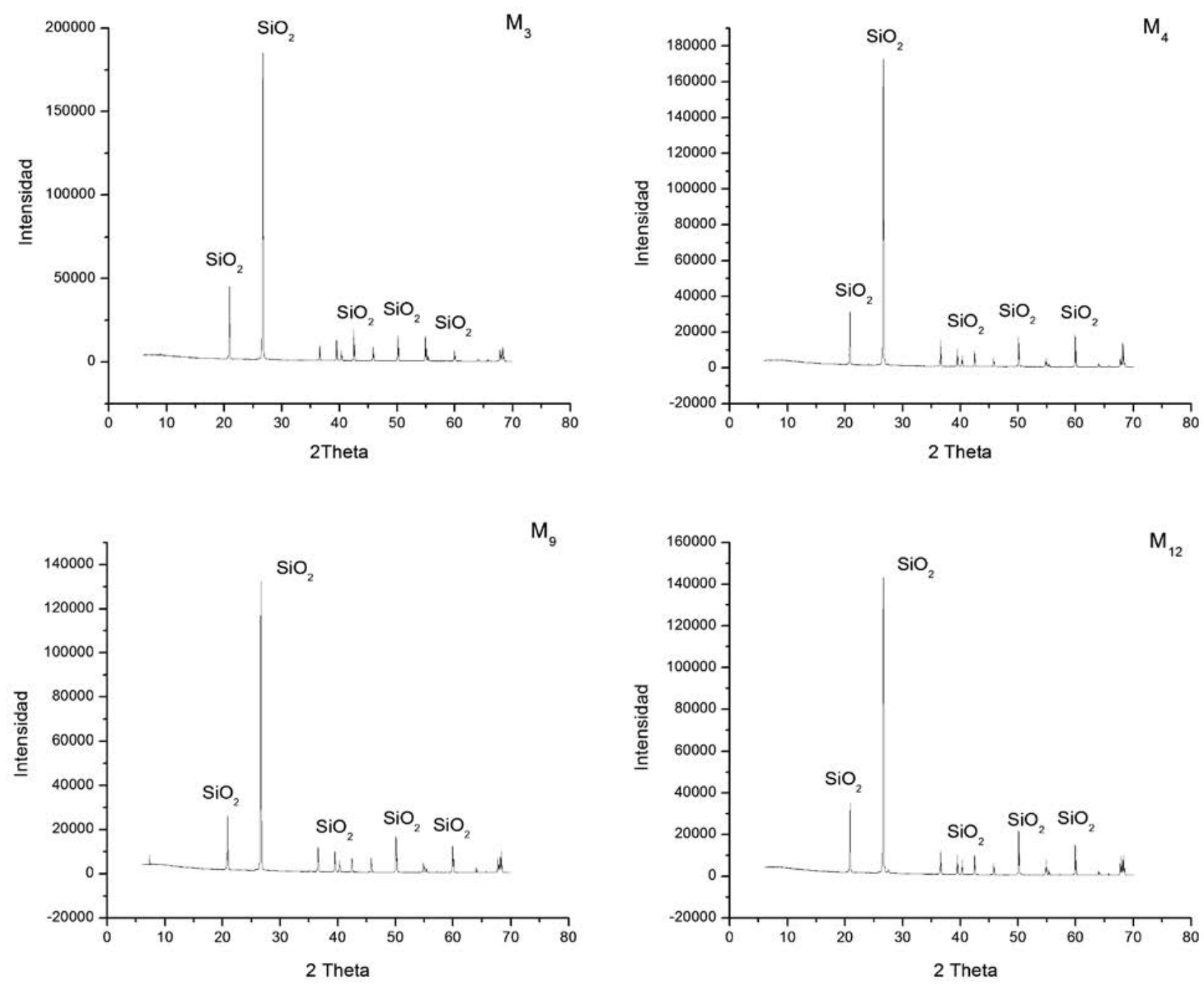

Figura 4. Difractogramas de las muestras de cuarzo 3, 4, 9 y 12 . Se observa las intensidades perteneciente al mineral.

Fuente: elaboración propia. 
En los difractogramas se observan diferentes picos de intensidades de rayos $\mathrm{X}$ difractados por la muestra de cuarzo. El valor más intenso se toma como 100, se toman los otros dos picos más intensos y por regla de tres se halla la intensidad relativa $\left(\mathrm{I} / \mathrm{I}_{0}\right)$. Los datos son comparados con la tarjeta 33116 de la base de datos PDF-2 Power Diffraction File, International Center Diffraction. Los resultados se resumen en la tabla 5 .

Para calcular el tamaño de los cristalitos se utilizó la ecuación de Scherrer. Este método consiste en extraer la información del tamaño del cristalito. Si la red cristalina está libre de deformación y los cristales predominan de manera general en las muestras, entonces se puede estimar el tamaño promedio del cristal empleando la ecuación (3). Los resultados se resumen en la tabla 6 .

$$
\beta=\frac{\lambda \times k}{F W H M \times \cos \theta}
$$

Donde,

$\beta$ : es el tamaño promedio de cristal.

$k$ : es el factor de forma del cristal y su valor es de 0,89 .

$\lambda$ : es la longitud de onda de la radiación utilizada $\left(\lambda_{\mathrm{Cu}}\right)$, que es de 1,5406.

$\theta$ : es la posición del pico de difracción.

FWHM: es el ancho a la altura media del pico de difracción de la muestra.

Tabla 5. Distancia entre los planos cristalográficos y valores de intensidad relativa de los picos l/I0 de las muestras de cuarzo

\begin{tabular}{|c|c|c|c|c|c|}
\hline & Angulo $2 \theta$ & $\begin{array}{c}\text { Distancia } \\
\text { nanómetros }(\mathrm{nm})\end{array}$ & $I / I_{0}$ & $\begin{array}{c}\text { Distancia } \\
\text { nanómetros }(\mathrm{nm}) *\end{array}$ & $\mathrm{I} / \mathrm{I}_{0}{ }^{*}$ \\
\hline \multirow{3}{*}{$M_{3}$} & $26,61^{\circ}$ & 0,335 & 100 & 0,334 & 100 \\
\hline & $20,851^{\circ}$ & 0,426 & 24,41 & 0,424 & 22 \\
\hline & $50,158^{\circ}$ & 0,182 & 8,71 & 0,181 & 10 \\
\hline \multirow{3}{*}{$M_{4}$} & $26,614^{\circ}$ & 0,335 & 100 & 0,334 & 100 \\
\hline & $20,856^{\circ}$ & 0,426 & 18,34 & 0,424 & 22 \\
\hline & $50,119^{\circ}$ & 0,182 & 10,01 & 0,181 & 10 \\
\hline \multirow{3}{*}{$M_{9}$} & $26,618^{\circ}$ & 0,335 & 100 & 0,334 & 100 \\
\hline & $20,856^{\circ}$ & 0,426 & 19,79 & 0,424 & 22 \\
\hline & $50,132^{\circ}$ & 10,82 & 12,8 & 0,181 & 10 \\
\hline \multirow{3}{*}{$M_{12}$} & $26,618^{\circ}$ & 0,335 & 100 & 0,334 & 100 \\
\hline & $20,849^{\circ}$ & 0,426 & 24,57 & 0,424 & 22 \\
\hline & $50,132^{\circ}$ & 0,182 & 10,27 & 0,181 & 10 \\
\hline
\end{tabular}

* PDF-2 Power Diffraction File, International Center Diffraction.

Fuente: elaboración propia.

Tabla 6. Tamaño de los cristalitos de cuarzo

\begin{tabular}{ccc}
\hline & Ángulo 20 & $\begin{array}{c}\text { Tamaño del cristal } \\
\text { nanómetros (nm) }\end{array}$ \\
\hline$M_{3}$ & $26,61^{\circ}$ & 29,5630 \\
\hline$M_{4}$ & $26,614^{\circ}$ & 28,2162 \\
\hline$M_{9}$ & $26,618^{\circ}$ & 28,2169 \\
\hline$M_{12}$ & $26,618^{\circ}$ & 28,2169 \\
\hline
\end{tabular}

Fuente: Elaboración Propia. 


\section{CONCLUSIONES}

Con la caracterización del cuarzo del municipio de La Playa de Belén se encontró, por medio de las tres técnicas utilizadas y la determinación de la densidad, que el mineral es $100 \%$ puro, que lo categoriza como cuarzo de alta pureza, al estar por encima de 99,95\% de composición de $\mathrm{SiO}_{2}$.

Con el estudio realizado se establecieron las características del afloramiento de cuarzo del municipio, tales como la composición química elemental, el porcentaje de los elementos, la morfología y el comportamiento térmico con el cambio de temperatura.

\section{FINANCIAMIENTO}

Este trabajo se llevó a cabo en el marco del proyecto "Desarrollo de un sistema de monitoreo cardiaco vestible mediante el uso de nanosensores de base textil reforzados con nanotubos de carbono", el cual fue aprobado por el Sistema General de Regalías en el año 2010. La investigación realizada tiene como objetivo principal realizar la caracterización específica de cuarzos en una zona del país, de gran interés para futuras explotaciones y exploraciones mineras, que posibilite el uso de este material en el desarrollo de aplicaciones biomédicas.

\section{REFERENCIAS}

Alfaro, M. (2002). Introducción al muestreo minero. Santiago de Chile: Instituto de Ingenieros de Minas de Chile.

Alipour, V., \& Denoyel, R. (2011). Characterization of wettability alteration of calcite, quartz and kaolinite: Surface energy analysis. Colloids and Surfaces A: Physicochem. Eng. Aspects , 98-108.

Anderson, J. (1998). Ciencia de los materiales (2 ed.). LIMUSA, S.A. México
Cornellis, K., Cornelius, K., \& Hurbult, S. y. (1996). Manual de minerología (4 ed.). Reverte, S.A.

Dana, \& Hurlbut. (1960). Manual de minerologia de dana (2 ed.). Barcelona-Buenos Aires-México: REVERTÉ, S.A.

Hilman, A., Ryder, K., Zaleski, C., Ferreira , V., Beasley, C., \& Vieil, E. (2014). Application of the combined electrochemical quartz crystalmicrobalance and probe beam deflection technique indeep eutectic solvents. Electrochimica Acta, 134(1) 42-51.

Holmes, A. (1980). Geologia FÍsica. Edicciones Omega $S . A$.

Hyodo, A., Kozdon, R., Pollington, A. D., \& Valley, J. W. (2014). Evolution of quartz cementation and burial history of the Eau Claire Formation based on in situ oxygen isotope analysis of quartz overgrowths. Chemical Geology , 384(1),168-180.

Leal, P., Cortona, O., \& Pagan, F. J. (2005). Vetas de cuarzo al sur de Santiago del Estero. Asociación Geológica de Argentina, 389-401.

Mendoza, H. H., Baron Gonzalez, A. J., \& Parra Vargas, C. A. (2007). Caracterización composicional de sílice de Ramiriquí Boyacá. Sciencia et Technica , 34(1), 647-651.

NMX-L-159-SCFI. (2003). Exploracion del petroleo-barita empleada en fluidos de perforacion, terminacion y mantenimiento de pozos petroleros-especificaciones y metodos de prueba. Ciudad de México: Normas Técnicas Mexicanas.

Rashad, A. M. (2012). A preliminary study of autoclaved alkali-activated slag blended with quartz powder. Construction and Buildin Materials, 33(1), 70-77.

Shackelford, J. (2010). Introducción a la ciencia de materiales para ingenieros. Pearson Educación, 6ta ed. Madrid.

Veranes, Y. (2003). Caracterización del cuarzo del yacimiento "El Cacahual" para la preparación de resinas compuestas. Revista Cubana de Quimica, 15 (2), 81-88.

\section{(c) $(1) \Theta \Theta$}


